\title{
Machine Learning and Artificial Intelligence in Neurocritical Care: a Specialty-Wide Disruptive Transformation or a Strategy for Success
}

\author{
Fawaz Al-Mufti ${ }^{1,2,3} \cdot$ Michael Kim $^{1} \cdot$ Vincent Dodson ${ }^{4} \cdot$ Tolga Sursal $^{1} \cdot$ Christian Bowers $^{1} \cdot$ Chad Cole $^{1}$. \\ Corey Scurlock ${ }^{5,6} \cdot$ Christian Becker $^{5,7} \cdot$ Chirag Gandhi $^{1} \cdot$ Stephan A. Mayer ${ }^{8}$
}

Published online: 13 November 2019

(C) Springer Science+Business Media, LLC, part of Springer Nature 2019

\begin{abstract}
Purpose of Review Neurocritical care combines the complexity of both medical and surgical disease states with the inherent limitations of assessing patients with neurologic injury. Artificial intelligence (AI) has garnered interest in the basic management of these complicated patients as data collection becomes increasingly automated.

Recent Findings In this opinion article, we highlight the potential AI has in aiding the clinician in several aspects of neurocritical care, particularly in monitoring and managing intracranial pressure, seizures, hemodynamics, and ventilation. The model-based method and data-driven method are currently the two major AI methods for analyzing critical care data. Both are able to analyze the vast quantities of patient data that are accumulated in the neurocritical care unit.

Summary AI has the potential to reduce healthcare costs, minimize delays in patient management, and reduce medical errors. However, these systems are an aid to, not a replacement for, the clinician's judgment.
\end{abstract}

Keywords Multimodality monitoring $\cdot$ Artificial intelligence $\cdot$ Neurocritical care $\cdot$ Closed-loop system

\section{Introduction}

Neurocritical care combines the complexity of both medical and surgical disease states with the inherent limitations of assessing patients with neurologic injury. Multimodality monitoring (MMM) allows the neurocritical care clinician to collect vast amounts of data including intracranial pressure (ICP), electroencephalograms (EEGs), hemodynamics, ventilation, body temperature, serial neurological examinations, fluid intake-output, and other neurophysiologic parameters [1]. MMM has transformed care by automating and centralizing patient data collection, allowing artificial intelligence (AI) to utilize this information and manage basic patient care. Recent advances have made it possible for AI to move from the experimental realm into the actual clinical setting in the intensive care unit (ICU). These AI systems that demonstrate ambient intelligence can interact with humans and are embedded, adaptive, personalized, context aware, and anticipatory. These traits allow AI systems to continuously monitor and treat neurocritical care patients in real time. Early signs of neurological deterioration could be detected more promptly and appropriate measures taken more quickly, thereby

This article is part of the Topical Collection on Critical Care

Fawaz Al-Mufti

fawazalmufti@outlook.com

1 Departments of Neurosurgery, Westchester Medical Center at New York Medical College, Valhalla, NY, USA

2 Departments of Neurology, Westchester Medical Center at New York Medical College, Valhalla, NY, USA

3 Neuroendovascular Surgery and Neurocritical Care Attending, Westchester Medical Center at New York Medical College, 100 Woods Road, Macy Pavilion 1331, Valhalla, NY 10595, USA
4 Department of Neurosurgery, New Jersey Medical School, Rutgers University, Newark, NJ, USA

eHealth Center, Westchester Medical Center Health Network, Valhalla, NY, USA

6 Departments of Anesthesiology, Westchester Medical Center at New York Medical College, Valhalla, NY, USA

7 Departments of Internal Medicine, Westchester Medical Center at New York Medical College, Valhalla, NY, USA

8 Department of Neurology, Henry Ford Health System, Detroit, MI, USA 
improving patient outcomes. Furthermore, AI could reduce costs and help patients in areas where neurocritical care is not available [2]. In this article, we review the promise of AI and its future potential directions in shaping neurocritical care.

\section{Promise of Artificial Intelligence}

AI systems have significantly advanced in the analysis of high-resolution neurocritical care data and the associated algorithmic decision-making [2]. Furthermore, iterative analysis of updated data creates a closed-loop AI system that monitors parameters, treats patients, and then modifies that treatment based on updated parameter data. Ultimately, the AI can manage patient care in real time without any clinician input. The combination of data analysis and decision-making creates the potential for an AI system to manage the minutiae of neurocritical patient management with minimal or no supervision, freeing the clinician to focus attention elsewhere [3-5]. Some potential parameters include anesthetics/analgesics, antiepileptic drugs (AED), blood pressure, glucose, fluids/electrolytes, neuromuscular blockade, and ventilator settings [6-17]. In this article, we discuss commonly measured parameters in neurocritical care, the innovations that allow AI to analyze patient data and manage these parameters, and the future potential of AI in the clinician's armamentarium.

\section{Bioinformatics, Algorithms, and Al Decision-making in Neurocritical Care}

Data analysis and decision-making are two features that are absolutely essential for an AI with ambient intelligence to function. In order to effectively assist the clinician in patient management, the AI must be able to parse through the large amounts of physiological data gathered by MMM, interpret the information, and then make complex neurocritical care decisions. The model-based methods and machine learning (data driven) methods are the two overarching general approaches taken when analyzing critical care data. The first model-based method utilizes a model constructed on our understanding of a system, and parameters are fed into that model to generate a predicted outcome. In clinical practice, this approach attempts to create a patient model that integrates various factors together and treatment then pushes the patient towards a favorable physiologic state rather than correct a specific physiological parameter $[18,19]$. The other machine learning method uses algorithms generated by prior data and outcomes to predict future outcomes based on unseen data. Both approaches use several methods to generate an outcome.

One such method is the dynamic systems model, which attempts to describe the many interactions in a system using classic physical mechanics. One example has been created by Ursino et al., in which he modeled the relationship between cerebral perfusion pressure, cerebral hemodynamics, cerebral vascular reserve, and autoregulation [20]. However, one potential problem with dynamic systems models is that they generally assume each component contributes linearly to the modeled biological system. However, real biological systems are typically nonlinear, so small changes in variables can potentially result in enormous downstream results [21].

Another method that can be used with model-based or machine learning systems is Bayesian inference, which uses Bayes rules to estimate diagnostic states of patients. This technique generates different diagnostic states and then assigns a probability of it being the patient's true state based on empirical data [22]. A best estimate of the patient's diagnostic state is made after each probability is weighed using Bayes rule. Furthermore, a dynamic Bayesian network can predict the transition from one diagnostic state to another [23]. The Avert-IT project by BrainIT is a real world example of a Bayesian network in neurocritical care [24]. It uses clinical, physiologic, and demographic data from traumatic brain injury (TBI) patients to create a prediction index of the occurrence of hypotension following TBI.

Machine learning systems use two types of learning algorithms, supervised or unsupervised. These algorithms use prior data and outcomes to predict future outcomes using unseen data. In supervised learning, the model is loaded with labeled data which is then used to predict an outcome. Outcomes are produced either by regression (predicting trends using previously labeled data) or by classification (as in decision tree analyses). In unsupervised learning, the system uses unlabeled data to identify patterns within the data itself. Many physicians are familiar with the decision tree analysis method as they are exposed to it during their training when using flowcharts to "work up" conditions. Complex multivariate data begins at the highest point and is then grouped into descending trees based on different factors (i.e., disease type, sex, age) [25]. These trees are then similarly subdivided using different factors. Ultimately, an endpoint can be examined (i.e., percentage success of outcome from a certain procedure) and then correlated with the parameters that were used to create that tree. An example could be "a 25\% success rate of fluid resuscitation in hemorrhagic hypotensive men over 85 " vs. "a $75 \%$ success rate of fluid resuscitation in hemorrhagic hypotensive women under 35 ".

Artificial neural networks are a particularly sophisticated form of supervised learning. These complex AI systems apply iterative learning to successfully accomplish multivariate nonlinear analysis and multifactorial classification. They may also discover patterns and sophisticated data relationships. The work of Vath et al. is one example of the clinical usage of artificial neural networks, which used clinical and neuromonitoring data to predict TBI outcomes [26]. Another example is the neural network developed by Cohen et al. It identifies clusters of physiological data in trauma patients in 
specific states by utilizing hierarchical clustering, a dimension reduction technique originally used in genomics [27]. This network is classified as unsupervised learning because it was able to identify and correlate data where the outcome was not known in a specific state and thereby discover new associations between different parameters. It correlated patient states (such as risk of infection, multiorgan failure, and death) with physiological data to identify prognostic patterns too complex for traditional techniques. It generated a dendrogram and a heat map to demonstrate "clusters" of physiologic data that were correlated with specific states in a visually apparent manner.

\section{Integrating Artificial Intelligence into Telehealth Platforms}

Increasing critical care provider shortages combined with inhomogeneities in provider distribution have resulted in increasing use of telemedicine applications in critical care including neurocritical care (telemedicine-supported ICU or tele-ICU) [28]. In the process of using this technology to enhance patient care, we have learned that, if implemented correctly, it can reduce length of stay and improve mortality [29, 30]. The need for tele-ICUs to be fully integrated with the bedside clinical care systems also provides the additional advantage of real-time interfacing and archiving of large clinical datasets across different platforms and through an integrated tele-ICU software system. The tele-ICU care delivery and enhancement model operates through small groups of expert telemedicine care providers who affect large numbers of patients. This enables tele-ICU-based clinical care standardization initiatives across large numbers of patients. The tele-ICU model has been shown to improve clinical outcomes through the development, implementation, and continuous improvement of AI-aided algorithmic clinical decision support systems [31].

As an example, tele-ICU services have been found to be associated with increased adherence to best practice guidelines $[32,33]$. A cornerstone of tele-ICU support is the early recognition of clinical deterioration and crisis prevention. Numerous algorithmic clinical decision support systems at increasing stages on the AI spectrum have been created for this purpose [34-36]. Taking this a step further, AI-supported algorithms also have made their way into capacity management, for example, by algorithmically providing real-time discharge readiness assessments with high discrimination for ICU mortality (AUROC 0.862) [37].

In the setting of increasing cost pressures and decreasing financial margins, optimizing patient throughput ("right patient, right bed, right time") has become more and more important. Integrating AI-supported surge predictive functions and throughput management functions into a comprehensive logistics center for hospitals and healthcare networks has been shown to increase capacity, improve patient outcomes, and increase financial contribution margins [37].

All AI approaches need large ideally multicenter or even international high-quality and granular datasets to develop and validate any AI application. Examples for such databases are the MIMIC III database and the eICU Collaborative Research Database [38]. Easy access to these datasets and collaborative approaches between data scientists and clinicians have spurned further research into AI support for critical care, as evidenced by recent publications $[38,39]$.

As an example of the promise of this approach, a recent study, targeting sepsis as a gigantic challenge on multiple levels, developed and validated a reinforcement learning agent (AI clinician) addressing fluid and vasoactive agent management decisions as crucial elements of acute management related to patient outcomes. The AI clinician reliably outperformed human clinicians in fluid and hemodynamic management, illustrating the potential for AI clinical decision support tools [40].

\section{Future Directions}

\section{Intracranial Pressure}

ICP is the most commonly monitored parameter in neurocritical care because it is highly predictive of mortality [41, 42]. Increased ICP can lead to impaired cerebral perfusion, brain tissue hypoxia, and subsequent infarction, thereby worsening secondary neurological injury and leading to eventual mortality. MMM can continuously record ICP, which allows the physician to be more responsive to adverse changes. AI can take advantage of the continuous monitoring to optimize treatment regimens in real time.

Some applications of AI for monitoring ICP already exist, albeit in experimental phases. One example is the forecast algorithm developed by Zhang et al., which predicts future mean ICP, enabling clinicians to identify dangerous trends in ICP early and potentially modify treatments to offset anticipated harmful increases in ICP [43]. This kind of technology would obviously be beneficial for patients within the ICU.

Other new approaches for analyzing ICP compares other physiologic parameters with ICP in real time. One such parameter is the pressure reactivity index (PRI), which determines if the patient is adequately maintaining cerebral pressure autoregulation by analyzing the trend between ICP and mean arterial pressure. This parameter has successfully been used in patients with TBI and in patients with intracerebral hemorrhage [44, 45]. Another parameter is ICP variability. ICU monitors typically display the latest ICP measurement, but AI systems that can calculate the variability of ICP over a 
given time may be more useful clinically since reduced ICP variability is associated with a poorer outcome [46, 47].

While AI has made advancements in data analysis in predicting future ICP trends and variability, the next logical step would be automating treatment in direct response to elevated ICP. With the continuous monitoring of ICP offered by MMM, an AI system could be programmed to automatically administer mannitol once it detects persistent elevation of ICP greater than $20 \mathrm{mmHg}$ for a pre-specified period of time assigned by the clinician. As the mannitol is being administered, the AI can sync to the electronic medical records and obtain data from the patient's blood work so that the serum osmolality does not rise about $320 \mathrm{mmol}$. If the ICP remains elevated despite the diuretic effect of mannitol, the AI could switch to treatment with hypertonic saline [48]. The urinary losses within this treatment period could be recorded and replaced using an automated fluid management system. The entirety of this process could be kept within a closed-loop system with minimal input from the clinician. The management of elevated ICP currently requires frequent checks on the patient by the ICU team. While AI cannot replace the vigilance of this team, the centralization of data and the automation of management would increase the probability that sudden elevations in ICP are addressed more promptly and efficiently.

\section{Seizures}

Another potential application of AI in neurocritical care includes the detection of seizures and the management of AEDs. Real-time computer-assisted analysis of EEGs has previously been shown to be able to classify seizures by type. An EEG classification system created by Cloostermaans et al. was able to categorize EEG activity into one of eight types: isoelectric, low voltage, burst suppression, generalized periodic discharge, seizure activity, slowed activity, artifact, and normal [49]. Artificial neural networks have also been used to detect seizure activity $[50,51]$. These computerized systems of detecting seizure activity can assist those who do not specialize in reading EEGs to manage patients with seizures, expediting treatment. Furthermore, this extends the reach of neurocritical care where epileptologists may not be available.

In addition to classifying EEG activity, AI can also automate the administration of AEDs. The current guidelines for treating status epilepticus require continuous EEG monitoring and titrating AEDs depending on the monitored epileptiform activity. Similar to monitoring ICP, blood pressure, and respiratory status, the role of $\mathrm{AI}$ in treating seizure activity would be to reduce the amount of time between the adverse event and administration of treatment. The AI system could function in a closed-loop system, constantly analyzing the EEG and titrating the AED infusion to the cessation of epileptiform activity as collected by EEG in a manner more precise than is currently possible [52].

\section{Blood Pressure}

Blood pressure management in the ICU is especially important as sudden changes can lead to permanent damage of several organs [53]. Optimal blood pressure management requires careful monitoring. The time frame in which the hypertension develops (urgency vs. emergency) and the degree of hypertension (moderate vs. severe) are important considerations. Since this data is already commonly collected in the ICU, an AI system can generate an automated response to administer an antihypertensive agent to achieve a blood pressure goal as assigned by the clinician. Furthermore, depending on the nature of a patient's hypertensive event, the AI could make suggestions as to which medication to administer. For example, if a patient develops tachycardia and/or arrhythmias, the AI might suggest beta-blockers because it algorithmically deduces an increased adrenergic tone in the patient. If the AI detects a concurrence of elevated blood pressure and increased ICP, it may once again suggest beta-blockers, but then warn against using vasodilators for it would be programmed to calculate cerebral perfusion pressure. It would advise against lowering blood pressure at the expense of decreasing cerebral perfusion. The physician is able to choose a treatment option based on the system's suggestions, and the AI is once again able to function in a closed-loop manner by continuing to monitor the blood pressure's response to the treatment given. For example, if the clinician wished that the blood pressure should not drop more than $20 \%$ within the first hour, the AI could continually adjust medication infusion rates to ensure blood pressure does not fall below this threshold [54].

\section{Ventilation}

The monitoring of a patient's respiratory status is important in neurocritical care as many patients with neurologic injury have an impaired respiratory drive [55]. Furthermore, inducing hyperventilation with subsequent hypocarbia is one way to manage elevated ICP in a patient [56]. These patients also often require positive end-expiratory pressure (PEEP) to improve their oxygenation status. However, while important for maintaining oxygenation, PEEP may contribute to increased ICP [57]. Therefore, the data from a patient's respiratory status must be analyzed with the patient's ICP. In an acute episode of intracranial hypertension, hyperventilation can be used to cause a drop in $\mathrm{PaCO}_{2}$, resulting in cerebral vasoconstriction and thereby reducing ICP. However, this is generally not recommended for prolonged management as the vasoconstriction can contribute to decreased cerebral blood flow, hypoxia, and even ischemia. Furthermore, the vasoconstrictive effect of hyperventilation has been shown to last less than $24 \mathrm{~h}$, after 
which the cerebral vasculature equilibrates with the new $\mathrm{PaCO}_{2}$, causing vessels to re-dilate with a subsequent rebound elevation in ICP [58]. In the future, it is easy to fathom that these interventions can be monitored and managed by AI systems since MMM already collects the necessary data including oxygen saturation, $\mathrm{PaCO}_{2}$, PEEP, and ICP. The AI could titrate the ventilator and respiratory settings in direct response to the parameters gathered, acutely responding to elevations in ICP. However, given the nuances and limitations in ventilator management in the neurocritical care patient, this automated system would require careful oversight by the intensive care team.

As discussed, one drawback of utilizing hyperventilation for ICP management is that it could induce cerebral ischemia [56]. Therefore, an ideal AI system would also incorporate data accumulated from continuous brain tissue oxygenation monitoring when managing ventilator and respiratory settings. The two main invasive methods to continuously monitor brain tissue oxygenation are intraparenchymal oxygen monitoring and jugular bulb oximetry $[59,60]$. In states of cerebral ische$\mathrm{mia}$, the brain increases oxygen extraction via a compensatory mechanism, reducing the jugular bulb venous oxygen saturation. Jugular bulb oximetry is useful because this increase in cerebral oxygen extraction occurs more than $24 \mathrm{~h}$ before the advancement to symptomatic cerebral vasospasm [61]. Similar to other parameters discussed, the physiologic data collected by MMM allows the AI system to automatically adjust ventilator settings in states of cerebral ischemia and then monitor the effects of the intervention. Ventilatory control in neurocritical care patients requires a fine balance between maintaining ICP and oxygenation. An AI can manage this balance in real time, reducing the time needed to make the necessary adjustments for a patient.

\section{Limitations}

AI technologies have great potential for assisting future neurocritical care physicians. However, significant hurdles remain before they can be used routinely in the ICU. One of the most significant challenges is creating adequate regulatory mechanisms to ensure the patients under the care of an AI are safe and protected. When an AI independently decides to intervene on a patient and causes an adverse event, who is then held accountable? Furthermore, patient autonomy might be challenged because the AI system can administer care in an automated fashion without involving the patient in the decision-making process. The patient-doctor relationship can become more "distant" as these AI systems take over more of the basic patient management. As this technology becomes more widespread, these challenges need to be addressed before an AI can play any role in patient care.

\section{Conclusion}

As MMM progresses and patient data continues to become increasingly automated and centralized, patient care will require ever more integration and analysis of multiple parameters. AI systems currently are suited to analyze the vast amounts of data, offering clinicians another tool in their armamentarium to manage these complex patients. The next goal in advancing neurocritical care is to develop AI systems that can function in closed loop systems, analyzing the data and titrating treatments in real time, reducing possible delays in patient care and optimizing the clinician's time. However, one must be cognizant and remember that these systems are in place to aid and not replace the clinician. The clinician is ultimately responsible for the patient's care.

Acknowledgments The editors would like to thank Dr. John Brust for taking the time to review this manuscript.

\section{Compliance with Ethical Standards}

Conflict of Interest Fawaz Al-Mufti, Michael Kim, Vincent Dodson, Tolga Sursal, Christian Bowers, Chad Cole, Corey Scurlock, Christian Becker, Chirag Gandhi, and Stephan A. Mayer each declare no potential conflicts of interest.

Human and Animal Rights and Informed Consent This article does not contain any studies with human or animal subjects performed by any of the authors.

\section{References}

1. Wartenberg KE, Schmidt JM, Mayer SA. Multimodality monitoring in neurocritical care. Crit Care Clin. 2007;23(3):507-38. https:// doi.org/10.1016/j.ccc.2007.06.002.

2. Hanson CW 3rd, Marshall BE. Artificial intelligence applications in the intensive care unit. Crit Care Med. 2001;29(2):427-35.

3. Bennett CC, Hauser K. Artificial intelligence framework for simulating clinical decision-making: a Markov decision process approach. Artif Intell Med. 2013;57(1):9-19. https://doi.org/10. 1016/j.artmed.2012.12.003.

4. Uemura K, Sugimachi M. Automated cardiovascular drug infusion system to control hemodynamics. Adv Biomed Eng. 2013;2:32-7. https://doi.org/10.14326/abe.2.32.

5. Rinehart J, Liu N, Alexander B, Cannesson M. Review article: closed-loop systems in anesthesia: is there a potential for closedloop fluid management and hemodynamic optimization? Anesth Analg. 2012;114(1):130-43. https://doi.org/10.1213/ANE. 0b013e318230e9e0.

6. Tehrani FT. A closed-loop system for control of the fraction of inspired oxygen and the positive end-expiratory pressure in mechanical ventilation. Comput Biol Med. 2012;42(11):1150-6. https://doi.org/10.1016/j.compbiomed.2012.09.007.

7. Tehrani F, Rogers M, Lo T, Malinowski T, Afuwape S, Lum M, et al. A dual closed-loop control system for mechanical ventilation. J Clin Monit Comput. 2004;18(2):111-29.

8. Wysocki M, Brunner JX. Closed-loop ventilation: an emerging standard of care? Crit Care Clin. 2007;23(2):223-40, ix. https:// doi.org/10.1016/j.ccc.2006.12.011. 
9. Lellouche F, Brochard L. Advanced closed loops during mechanical ventilation (PAV, NAVA, ASV, SmartCare). Best Pract Res Clin Anaesthesiol. 2009;23(1):81-93.

10. Liu N, Chazot T, Hamada S, Landais A, Boichut N, Dussaussoy C, et al. Closed-loop coadministration of propofol and remifentanil guided by bispectral index: a randomized multicenter study. Anesth Analg. 2011;112(3):546-57. https://doi.org/10.1213/ANE. 0b013e318205680b.

11. Liu N, Chazot T, Genty A, Landais A, Restoux A, McGee K, et al. Titration of propofol for anesthetic induction and maintenance guided by the bispectral index: closed-loop versus manual control: a prospective, randomized, multicenter study. Anesthesiology. 2006;104(4):686-95.

12. Puri GD, Kumar B, Aveek J. Closed-loop anaesthesia delivery system (CLADS) using bispectral index: a performance assessment study. Anaesth Intensive Care. 2007;35(3):357-62.

13. Janda M, Simanski O, Bajorat J, Pohl B, Noeldge-Schomburg GF, Hofmockel R. Clinical evaluation of a simultaneous closed-loop anaesthesia control system for depth of anaesthesia and neuromuscular blockade*. Anaesthesia. 2011;66(12):1112-20. https://doi. org/10.1111/j.1365-2044.2011.06875.x.

14. Eleveld DJ, Proost JH, Wierda JMKH. Evaluation of a closed-loop muscle relaxation control system. Anesth Analg. 2005;101(3):75864. https://doi.org/10.1213/01.ane.0000167069.54613.50.

15. Cavalcanti AB, Silva E, Pereira AJ, Caldeira-Filho M, Almeida FP, Westphal GA, et al. A randomized controlled trial comparing a computer-assisted insulin infusion protocol with a strict and a conventional protocol for glucose control in critically ill patients. J Crit Care. 2009;24(3):371-8. https://doi.org/10.1016/j.jcrc.2009.05. 005.

16. Rinehart J, Lee C, Cannesson M, Dumont G. Closed-loop fluid resuscitation: robustness against weight and cardiac contractility variations. Anesth Analg. 2013;117(5):1110-8. https://doi.org/10. 1213/ANE.0b013e3182930050.

17. Kramer GC, Kinsky MP, Prough DS, Salinas J, Sondeen JL, HazelScerbo ML, et al. Closed-loop control of fluid therapy for treatment of hypovolemia. J Trauma. 2008;64(4 Suppl):S333-41. https://doi. org/10.1097/TA.0b013e31816bf517.

18. Buchman TG. Novel representation of physiologic states during critical illness and recovery. Crit Care. 2010;14(2):127. https://doi. org/10.1186/cc8868.

19. Buchman TG. Physiologic stability and physiologic state. J Trauma. 1996;41(4):599-605.

20. Ursino M, Lodi CA, Rossi S, Stocchetti N. Estimation of the main factors affecting ICP dynamics by mathematical analysis of PVI tests. Acta Neurochir Suppl. 1998;71:306-9.

21. Coveney PV, Fowler PW. Modelling biological complexity: a physical scientist's perspective. J R Soc Interface. 2005;2(4):267-80. https://doi.org/10.1098/rsif.2005.0045.

22. Ashby D. Bayesian statistics in medicine: a 25 year review. Stat Med. 2006;25(21):3589-631. https://doi.org/10.1002/sim.2672.

23. Peelen L, de Keizer NF, Jonge E, Bosman RJ, Abu-Hanna A, Peek $\mathrm{N}$. Using hierarchical dynamic Bayesian networks to investigate dynamics of organ failure in patients in the intensive care unit. J Biomed Inform. 2010;43(2):273-86. https://doi.org/10.1016/j.jbi. 2009.10.002

24. Stell A, Sinnott R, Jiang J, Donald R, Chambers I, Citerio G, et al. Federating distributed clinical data for the prediction of adverse hypotensive events. Philos Transact A Math Phys Eng Sci. 2009;367(1898):2679-90. https://doi.org/10.1098/rsta.2009.0042.

25. McQuatt A, Sleeman D, Andrews PJ, Corruble V, Jones PA. Discussing anomalous situations using decision trees: a head injury case study. Methods Inf Med. 2001;40(5):373-9.

26. Vath A, Meixensberger J, Dings J, Meinhardt M, Roosen K. Prognostic significance of advanced neuromonitoring after traumatic brain injury using neural networks. Zentralbl Neurochir. 2000;61(1):2-6.

27. Cohen MJ, Grossman AD, Morabito D, Knudson MM, Butte AJ, Manley GT. Identification of complex metabolic states in critically injured patients using bioinformatic cluster analysis. Crit Care. 2010;14(1):R10. https://doi.org/10.1186/cc8864.

28. Lilly CM, Zubrow MT, Kempner KM, Reynolds HN, Subramanian S, Eriksson EA, et al. Critical care telemedicine: evolution and state of the art. Crit Care Med. 2014;42(11):2429-36. https://doi.org/10. 1097/ccm.0000000000000539.

29. Becker CD, Fusaro MV, Scurlock C. Deciphering factors that influence the value of tele-ICU programs. Intensive Care Med. 2019;45(7):1046-51. https://doi.org/10.1007/s00134-019-055914.

30. Becker CD, Fusaro MV, Scurlock C. Telemedicine in the ICU: clinical outcomes, economic aspects, and trainee education. Curr Opin Anaesthesiol. 2019;32:129-35. https://doi.org/10.1097/aco. 0000000000000704.

31. Kindle RD, Badawi O, Celi LA, Sturland S. Intensive care unit telemedicine in the era of big data, artificial intelligence, and computer clinical decision support systems. Crit Care Clin. 2019;35(3): 483-95. https://doi.org/10.1016/j.ccc.2019.02.005.

32. Lilly CM, Cody S, Zhao H, Landry K, Baker SP, McIlwaine J, et al. Hospital mortality, length of stay, and preventable complications among critically ill patients before and after tele-ICU reengineering of critical care processes. JAMA. 2011;305(21):2175-83. https:// doi.org/10.1001/jama.2011.697.

33. Lilly CM, McLaughlin JM, Zhao H, Baker SP, Cody S, Irwin RS. A multicenter study of ICU telemedicine reengineering of adult critical care. Chest. 2014;145(3):500-7. https://doi.org/10.1378/chest. 13-1973.

34. Badawi O, Liu X, Hassan E, Amelung PJ, Swami S. Evaluation of ICU risk models adapted for use as continuous markers of severity of illness throughout the ICU stay. Crit Care Med. 2018;46(3):3617. https://doi.org/10.1097/ccm.0000000000002904.

35. Badawi O, Breslow MJ. Readmissions and death after ICU discharge: development and validation of two predictive models. PLoS One. 2012;7(11):e48758. https://doi.org/10.1371/journal. pone. 0048758 .

36. Escobar GJ, Turk BJ, Ragins A, Ha J, Hoberman B, LeVine SM, et al. Piloting electronic medical record-based early detection of inpatient deterioration in community hospitals. J Hosp Med. 2016;11(Suppl 1):S18-s24. https://doi.org/10.1002/jhm.2652.

37. Lilly CM, Motzkus C, Rincon T, Cody SE, Landry K, Irwin RS. ICU telemedicine program financial outcomes. Chest. 2017;151(2): 286-97. https://doi.org/10.1016/j.chest.2016.11.029.

38. Pollard TJ, Johnson AEW, Raffa JD, Celi LA, Mark RG, Badawi O. The eICU Collaborative Research Database, a freely available multi-center database for critical care research. Sci Data. 2018;5: 180178. https://doi.org/10.1038/sdata.2018.178.

39. McShea M, Holl R, Badawi O, Riker RR, Silfen E. The eICU research institute-a collaboration between industry, health-care providers, and academia. IEEE Eng Med Biol Mag. 2010;29(2):18-25. https://doi.org/10.1109/memb.2009.935720.

40. Komorowski M, Celi LA, Badawi O, Gordon AC, Faisal AA. The Artificial Intelligence Clinician learns optimal treatment strategies for sepsis in intensive care. Nat Med. 2018;24(11):1716-20. https:// doi.org/10.1038/s41591-018-0213-5.

41. Zoerle T, Lombardo A, Colombo A, Longhi L, Zanier ER, Rampini $\mathrm{P}$, et al. Intracranial pressure after subarachnoid hemorrhage. Crit Care Med. 2015;43(1):168-76. https://doi.org/10.1097/ccm. 0000000000000670.

42. Heuer GG, Smith MJ, Elliott JP, Winn HR, LeRoux PD. Relationship between intracranial pressure and other clinical variables in patients with aneurysmal subarachnoid hemorrhage. J 
Neurosurg. 2004;101(3):408-16. https://doi.org/10.3171/jns.2004. 101.3.0408.

43. Zhang F, Feng M, Pan SJ, Loy LY, Guo W, Zhang Z, et al. Artificial neural network based intracranial pressure mean forecast algorithm for medical decision support. Conf Proc IEEE Eng Med Biol Soc. 2011;2011:7111-4.

44. Diedler J, Sykora M, Rupp A, Poli S, Karpel-Massler G, Sakowitz $\mathrm{O}$, et al. Impaired cerebral vasomotor activity in spontaneous intracerebral hemorrhage. Stroke. 2009;40(3):815-9. https://doi.org/10. 1161/strokeaha.108.531020.

45. Steiner LA, Czosnyka M, Piechnik SK, Smielewski P, Chatfield D, Menon DK, et al. Continuous monitoring of cerebrovascular pressure reactivity allows determination of optimal cerebral perfusion pressure in patients with traumatic brain injury. Crit Care Med. 2002;30(4):733-8.

46. Kirkness CJ, Burr RL, Mitchell PH. Intracranial pressure variability and long-term outcome following traumatic brain injury. Acta Neurochir Suppl. 2008;102:105-8.

47. Hornero R, Aboy M, Abasolo D, McNames J, Goldstein B. Interpretation of approximate entropy: analysis of intracranial pressure approximate entropy during acute intracranial hypertension. IEEE Trans Biomed Eng. 2005;52(10):1671-80. https://doi.org/ 10.1109/tbme.2005.855722.

48. Rangel-Castillo L, Gopinath S, Robertson CS. Management of intracranial hypertension. Neurol Clin. 2008;26(2):521-41. https:// doi.org/10.1016/j.ncl.2008.02.003.

49. Cloostermans MC, de Vos CC, van Putten MJ. A novel approach for computer assisted EEG monitoring in the adult ICU. Clin Neurophysiol. 2011;122(10):2100-9. https://doi.org/10.1016/j. clinph.2011.02.035.

50. Guo L, Rivero D, Dorado J, Rabunal JR, Pazos A. Automatic epileptic seizure detection in EEGs based on line length feature and artificial neural networks. J Neurosci Methods. 2010;191(1):101-9. https://doi.org/10.1016/j.jneumeth.2010.05.020.

51. Pravin Kumar S, Sriraam N, Benakop PG, Jinaga BC. Entropies based detection of epileptic seizures with artificial neural network classifiers. Expert Syst Appl. 2010;37(4):3284-91. https://doi.org/ 10.1016/j.eswa.2009.09.051.

52. Brophy GM, Bell R, Claassen J, Alldredge B, Bleck TP, Glauser T, et al. Guidelines for the evaluation and management of status epilepticus. Neurocrit Care. 2012;17(1):3-23. https://doi.org/10. 1007/s12028-012-9695-z.

53. Aggarwal M, Khan IA. Hypertensive crisis: hypertensive emergencies and urgencies. Cardiol Clin. 24(1):135-46. https://doi.org/10. 1016/j.ccl.2005.09.002.

54. Salgado DR, Silva E, Vincent J-L. Control of hypertension in the critically ill: a pathophysiological approach. Ann Intensive Care. 2013;3:17. https://doi.org/10.1186/2110-5820-3-17.

55. Borel C, Briegel J, Hanley DF. Respiratory management in neurological critical care: basics and techniques of artificial ventilation. In: Neurocritical Care. Springer; 1994; pp 139-156.

56. Stocchetti N, Maas AI, Chieregato A, van der Plas AA. Hyperventilation in head injury: a review. Chest. 2005;127(5): 1812-27. https://doi.org/10.1378/chest.127.5.1812.

57. Caricato A, Conti G, Della Corte F, Mancino A, Santilli F, Sandroni $\mathrm{C}$, et al. Effects of PEEP on the intracranial system of patients with head injury and subarachnoid hemorrhage: the role of respiratory system compliance. J Trauma. 2005;58(3):571-6.

58. Muizelaar JP, van der Poel HG, Li ZC, Kontos HA, Levasseur JE. Pial arteriolar vessel diameter and $\mathrm{CO} 2$ reactivity during prolonged hyperventilation in the rabbit. J Neurosurg. 1988;69(6):923-7. https://doi.org/10.3171/jns.1988.69.6.0923.

59. Ulrich CT, Fung C, Vatter H, Setzer M, Gueresir E, Seifert V, et al. Occurrence of vasospasm and infarction in relation to a focal monitoring sensor in patients after SAH: placing a bet when placing a probe? PLoS One. 2013;8(5):e62754. https://doi.org/10.1371/ journal.pone.0062754.

60. Kistka H, Dewan MC, Mocco J. Evidence-based cerebral vasospasm surveillance. Neurol Res Int. 2013;2013:256713-6. https:// doi.org/10.1155/2013/256713.

61. Heran NS, Hentschel SJ, Toyota BD. Jugular bulb oximetry for prediction of vasospasm following subarachnoid hemorrhage. Can J Neurol Sci. 2004;31(1):80-6.

Publisher's Note Springer Nature remains neutral with regard to jurisdictional claims in published maps and institutional affiliations. 